\title{
Kenneth M. Kensinger (1931-2010)
}

\section{Philippe Erikson}

\section{OpenEdition}

\section{Journals}

\section{Edición electrónica}

URL: https://journals.openedition.org/jsa/11859

DOI: 10.4000/jsa. 11859

ISSN: $1957-7842$

\section{Editor}

Société des américanistes

\section{Edición impresa}

Fecha de publicación: 20 diciembre 2011

Paginación: 388-396

ISSN: 0037-9174

\section{Referencia electrónica}

Philippe Erikson, «Kenneth M. Kensinger (1931-2010)», Journal de la Société des américanistes [En

línea], 97-2 | 2011, Publicado el 22 enero 2012, consultado el 03 septiembre 2022. URL: http:// journals.openedition.org/jsa/11859 ; DOl: https://doi.org/10.4000/jsa.11859 


\section{Kenneth M. KENSINGER (1931-2010)}

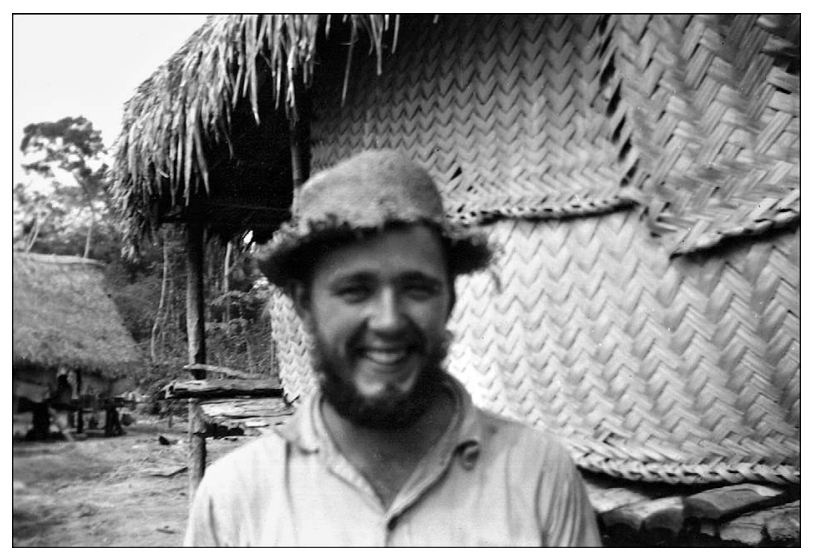

Kenneth M. Kensinger nous a quittés le 14 mai 2010. Le tonnerre a-t-il grondé, ce jour-là, dans le village cashinahua de Balta, au Pérou, où il aura vécu tant d'années ? Avec nukun haibu Kin (« notre ami Ken »), c'est en tout cas une figure légendaire de l'américanisme tropical qui disparait. Celle d'un homme qui rédigeait ses notes de terrain en cashinahua et qui, 25 ans après son retour aux États-Unis, rêvait encore dans cette langue ; celle d'un passionné du terrain, où il aura passé près de huit années de sa vie (84 mois entre 1955 et 1968 et, à nouveau, 8 mois en 1993-1994).

Né à Altoona (Pennsylvanie) dans un milieu strictement conservateur et rigoureusement religieux, Kensinger s'est pourtant très tôt intéressé à la diversité culturelle. Lecteur vorace et passionné, il s'est efforcé, dès la fin de son adolescence, de " read his way around the world », comme il le disait lui-même. Il n'a par la suite jamais dévié de cette voie. Les étagères de son bureau regorgeaient de livres en double exemplaire, destinés à ses visiteurs. À l'issue de notre première rencontre en 1987, outre ses œuvres complètes dédicacées " to a fellow explorer and panologist », Kenneth m'offrit deux ouvrages particulièrement révélateurs de ses goûts et de sa personnalité. L'un sur les croustillants rites d'initiation mélanésiens (Gilbert Herdt, éd., Rituals of manhood, 1982) et l'autre rédigé par l'un de ses mentors, Robert Murphy (Dialectical anthropology, 1974). Ses amis repartaient toujours de chez lui avec des confitures maison, des cassettes vidéo, des photos (comme celle le représentant sur le terrain reproduite ci-dessus) et, surtout, une multitude d'anecdotes et du baume au cœur.

La conjonction de son attrait pour l'exotique et de ses origines puritaines, associées à une immense générosité, l'entraînèrent logiquement à s'engager dans 
la carrière de missionnaire. En 1955, le Wycliffe Bible Translators/Summer Institute of Linguistics, qui semble avoir beaucoup recruté en Pennsylvanie ${ }^{1}$, l'affecta alors au Pérou, chez les Cashinahua. Récemment décimés par de terribles épidémies, ces derniers vivaient isolés au plus profond de la forêt, sur le rio Xapuya, dans une région où il aurait été impossible d'ouvrir une piste d'atterrissage. Ils acceptèrent toutefois de se regrouper autour de lui plus en aval, sur le Curanja, d'abord à Conta, puis à Balta. Il y travailla de nombreuses années, bientôt rejoint par d'autres missionnaires, Gail et Robert Cromack (ultérieurement remplacés par Susan et Richard Montag). Aujourd'hui encore, les Cashinahua du Pérou le voient comme une figure semi-légendaire : celle de l'homme qui accompagna leur rapprochement du monde des « Inka » (étrangers, Blancs); celle de l'homme qui donna son surnom à Grompes (ou Kundunpes, déformation de Krönprinz), leader charismatique de Balta, dont il rédigea dernièrement une émouvante nécrologie (Kensinger 2003a).

Loin d'être à sens unique, l'influence fut au contraire réciproque. À la différence d'autres missionnaires, Kensinger ne se fit pas construire de maison dans les arbres pour s'y isoler de ses ouailles. Passionné de chasse et de botanique, il vivait en immersion totale avec les Cashinahua. Il emmenait certes de la choucroute sur le terrain pour varier l'ordinaire et parce qu'elle ne suscitait guère de convoitises chez ses hôtes; mais il s'acclimata si bien à leur mode de vie qu'il finit par en perdre sa vocation et quitta le SIL en 1963. S'ensuivit, au milieu des années 1960, sa « (re)conversion » à l'ethnologie, qui lui permettait de poursuivre son œuvre tout en abandonnant la mission. Kensinger comparait volontiers sa trajectoire à celle de son frère jumeau Keith, resté aux États-Unis, devenu pasteur et qui, bien qu'ayant lui aussi vu sa foi décliner, ne changea pas d'orientation, convaincu qu'il était de l'utilité sociale de sa profession, de l'importance de l'aide qu'il pouvait apporter aux gens.

Revenu en Amérique du Nord, Kensinger reprit ses études à Philadelphie, où il obtint son Artium Magister en 1964. En dépit des centaines de pages rédigées à l'époque sous la direction de Ward Goodenough (un autre de ses héros intellectuels, avec Kenneth Pike), il ne parvint cependant jamais à achever sa thèse de doctorat. Sa réinsertion professionnelle s'effectua toutefois sans difficulté majeure. Son charisme, ses talents d'orateur, son immense érudition et son exceptionnelle expérience du terrain lui permirent d'occuper d'abord quelques postes temporaires à San Francisco State et Temple University (1966-1970), puis à Columbia (1970-1971), avant de se voir définitivement embaucher à Bennington College, dans le Vermont. Kensinger y passa les vingt-cinq dernières années de sa carrière et réussit à transformer ce très huppé Liberal Arts College, essentiellement tourné vers l'enseignement artistique, en haut-lieu de l'américanisme tropical.

La période de transition entre les deux grandes étapes de sa vie et de sa carrière aura toutefois été difficile. Pour toutes sortes de raisons, bien qu'il y soit resté en 
pensée jusqu'à la fin de sa vie, Kensinger avait pour ainsi dire totalement rompu les ponts avec le Pérou. Après son recrutement à Bennington, il n'y remit d'ailleurs les pieds qu'un quart de siècle plus tard. Apostat, il était naturellement en froid avec ses anciens confrères de la mission, qu'il ne revit brièvement qu'en $1991^{2}$. Il semble également que les relations avec les Cashinahua aient été compliquées, notamment par diverses collectes effectuées sur le terrain peu avant son départ définitif. Dans le cadre de ses multiples et fructueuses collaborations avec des anthropologues physiques et des muséographes (voir la liste des publications), de nombreux échantillons sanguins furent en effet prélevés, et d'importantes collections d'artisanat furent constituées ${ }^{3}$. On peut facilement imaginer les tensions que cela provoqua. L'intention était généreuse, puisqu'il s'agissait de contribuer à l'avancement de la science et à la diffusion du savoir, mais les effets secondaires, sur le plan émotionnel, furent désastreux. On ne peut que regretter que Kensinger n'ait jamais publié, à ce propos, de texte aussi lucide que son poignant : "“You killed my baby!" : The dilemmas of medical intervention during fieldwork ", qui raconte la gestion d'une tragique histoire de surdosage médicamenteux sur le terrain.

Il semble cependant qu'au retour du Pérou les difficultés les plus pénibles à surmonter aient été d'ordre personnel, la morosité et la démotivation ayant, de son propre aveu, longtemps affecté Kensinger. Pur produit d'une Amérique polarisée entre le puritanisme religieux et le post-modernisme hippie, Ken mit assez longtemps à trouver son équilibre entre les deux, notamment grâce à la psychanalyse. À l'évidence, les campus américains de la fin des années 1960 avaient de quoi dérouter quelqu'un qui, élevé dans le voisinage de familles amish, avait ensuite passé une dizaine d'années en Amazonie...

De toute cette époque, et pour résumer son parcours, Kensinger disait souvent que les Cashinahua l'avaient dé-converti du christianisme pour le convertir à l'anthropologie, mais que leurs hallucinogènes lui avaient ensuite ouvert les yeux sur la vanité du projet anthropologique lui-même. Au cours d'une vision provoquée par l'ayahuasca, aimait-il à raconter, il vit un immense mixeur, plus haut que lui, dans lequel tourbillonnaient les noms des plus grands ethnologues : Boas, Evans-Pritchard, Lévi-Strauss, Malinowski, etc. S'approchant du récipient pour voir ce qui s'y concoctait réellement, il dut se rendre à l'évidence : en réalité, tout cela n'était que de la merde...

Destin exceptionnel que celui de cet homme qui aura connu plusieurs carrières aussi différentes, aura su s'adapter et se faire apprécier dans des milieux aussi hétéroclites, mais qui ne s'en caractérisait pas moins, avant tout, par sa constance et sa fidélité. Ken prenait chaque jour son petit déjeuner au Blue Benn, désuète caravane en aluminium transformée en "diner » typique de la vieille Amérique. Il se plaignait même de souffrir d'un " withdrawal syndrom » s'il était trop longtemps privé des omelettes qu'on y servait. Bien qu'il ait pu rester quelque temps à Bennington après son départ en retraite en 1996, l'obligation qui 
lui fut finalement faite de quitter sa maison sur le campus fut pour lui une pénible épreuve. Malgré la brièveté de ses passages à Temple, Berkeley et Columbia, il y noua de durables liens d'amitié qui ne furent jamais rompus par la suite. Kensinger, qui aimait les chiens et le jardinage, aura été fidèle jusque dans le choix de ses supports de publication, publiant dans les mêmes revues à près de 30 ans d'intervalle!

Autant que par son œuvre, pourtant considérable, c'est par son charisme et ses talents d'animateur de la recherche que Kenneth Kensinger aura durablement marqué le milieu des amazonistes nord-américains. Respecté pour sa connaissance sans faille des Cashinahua, apprécié pour sa disponibilité, il incarnait la figure du sage patriarche, du modérateur par excellence. Les réunions qu'il organisait, chaque été, dans l'ambiance feutrée de Bennington College, étaient un des hauts lieux de la sociabilité des ethnologues de la côte est ${ }^{4}$. On y venait aisément en voiture depuis New York, Boston, voire Philadelphia ou Montréal, mais souvent aussi de plus loin - de Chicago, de Floride, de Californie, et même d'Amérique latine ou d'Europe -, pour s'entretenir en petit comité de l'actualité de la recherche amazoniste. Les sessions se déroulaient dans de confortables fauteuils, entourés de fleurs et de bols de fruits. "Is this a creative writing workshop? " demanda un jour une étudiante visiblement fascinée, entrée par erreur dans le salon où Emilienne Ireland présentait ses données sur la sorcellerie dans le Haut-Xingu... L'atmosphère était vraiment magique, et l'intérêt de telles réunions était tel que lorsque le départ en retraite de Kensinger y mit fin, la communauté américaniste décida de s'organiser en association professionnelle. Ainsi naquit SALSA (Society for the Anthropology of Lowland South America), dont les membres fondateurs reconnaissent tous leur dette intellectuelle à l'égard de Kensinger, et dont le site web héberge des pages spécifiquement dédiées à sa mémoire [ http://www.salsa-tipiti.org/Kensinger/kensingerportal.html].

Kensinger organisait également des événements très prisés à l'occasion de certains des Congrès Internationaux des Américanistes et s'assurait que chaque réunion de l'American Anthropological Association comprenne au moins une session amazoniste. Il y présentait des communications toujours admirables, même si les publications subséquentes laissaient parfois un goût d'inachevé. Elles étaient toujours trop brèves, surtout à l'aune de ce qu'on savait que l'auteur savait des Cashinahua. Il revenait souvent à d'autres le soin de peaufiner les thèmes qu'il avait ébauchés ${ }^{5}$. Cependant, Kensinger se montrait infatigable quand il s'agissait de publier les travaux d'autrui, et Bennington fut le siège de deux séries de publications qui auront marqué la recherche américaniste de la seconde moitié du $\mathrm{Xx}^{\mathrm{e}}$ siècle. En fin de carrière, l'importance de son rôle fut solennisée par le doctorat honoris causa qu'il reçut en 1999 de l'université d'Uppsala, à laquelle il légua sa bibliothèque et toutes ses archives personnelles.

Ken Ken, comme l'appelaient ses proches, aura vécu " The way real people ought to live ". Lorsque ses hôtes le quittaient, il avait l'habitude de leur offrir une 
généreuse collation et détestait qu'on lui dise merci. Sa mère n'avait pas lu Mauss, disait-il, mais elle aussi détestait qu'on la remercie, pressentant que c'était une manière de boucler trop vite une relation ${ }^{6}$. Les Cashinahua aussi donnaient de la nourriture à leurs visiteurs, poursuivait-il, mais non sans y trouver leur compte. Ils s'assuraient ainsi qu'avec assez de provisions pour au moins la moitié du chemin, ils ne risquaient pas de revenir puiser dans les réserves de leurs amphitryons! Généreusement pourvu par le destin, Kensinger a pu parcourir son prodigieux chemin jusqu'à sa destination finale. Le tonnerre a grondé à Balta.

\section{Notes}

1. Kensinger racontait volontiers qu'avant même qu'aucun des trois ne s'engage dans l'activité missionnaire, il avait été fiancé à une autre figure légendaire du SIL, mais qu'elle s'était finalement mariée avec un troisième (futur) membre de l'Institut... Une émouvante trace de ces histoires de mariage raté se retrouve dans sa toute première publication, un texte de 1963 consacré à la phonologie du cashinahua ; texte certes aussi austère que le suggère son titre, mais dont tous les exemples tournent autour de la question des déboires amoureux de l'auteur. La sexualité comptera ultérieurement parmi les thèmes récurrents de l'œuvre de Kensinger.

2. Kensinger revit certains de ses anciens compagnons du SIL en 1991 à l'occasion du symposium "Classic Panoan Topics in Light of Recent Research », co-organisé avec Barbara Keifenheim, avec l'aide (officieuse) de l'auteur de ces lignes, dans le cadre du 47th International Congress of Americanists, à New Orleans. S'y étaient retrouvés tant les missionnaires et les indigénistes que les ethnologues, les archéologues et les linguistes du monde entier œuvrant dans l'aire linguistique pano. Bien que l'événement ait rencontré un succès considérable, les actes ne furent jamais publiés.

3. Une longue interview filmée par Gordon Gray, en 2009, à l'université de Temple (voir filmographie) fournit de précieuses informations sur les objets cashinahua collectés dans les années 1960.

4. Ces réunions prenaient le relais de celles qu'organisait auparavant Robert Murphy, d'abord à Columbia University (New York), puis, lorsqu'il fut atteint de paralysie, à son domicile. Kensinger était assidu à ces « NYC movable feasts of Lowland South Americanists » (pour reprendre une expression de Janet Chernela).

5. Ses deux textes très novateurs sur les conceptions indigènes de l'inscription plurielle du savoir en plusieurs points du corps (1992a et 1994) ont par exemple permis à Cecilia McCallum de rédiger, en 1996, le bien plus détaillé : "The body that knows : from Cashinahua epistemology to a medical anthropology of Lowland South America ", Medical Anthropology Quarterly, 10 (3), pp. 342-372.

6. Tocqueville avait déjà noté que les habitants de Pennsylvanie ne détestaient rien plus que les remerciements ! De manière générale la modestie était, pour Kensinger, une vertu cardinale. Il excusait cependant volontiers la vantardise pathologique d'un autre missionnaire reconverti comme lui à l'ethnologie, en invoquant le passé douloureux de cet homme élevé dans une famille où, confondant humilité et humiliation, l'on rabrouait constamment les enfants d'une simple abréviation : "SPS » ( « self praise sucks »)... Le goût de Kensinger pour la simplicité se retrouvait jusque dans la sobriété des couvertures et la modestie des intitulés des deux séries de recueils qu'il publia à intervalles irréguliers depuis Bennington : "Working papers on South American Indians » (1979-1985) et " South American Indian studies » (1993-1998). Les articles (aujourd'hui disponibles en ligne) y étaient généralement trop courts, mais les thématiques particulièrement en phase avec l'actualité scientifique de l'époque. 


\section{PRINCIPALES PUBLICATIONS}

Une bibliographie exhaustive de Kenneth Kensinger est disponible en ligne sur le site du Journal [http://jsa.revues.org/index11859.html].

\section{OUVRaGeS}

1975 The Cashinahua of Eastern Peru, The Haffenreffer Museum of Anthropology, Brown University, Bristol, Rhode Island [édition scientifique : Jane Powell Dwyer ; 320 fig. ; avec les contributions, analysant les pièces de la collection Kensinger, de Phyllis Rabineau, Helen Tanner, Susan G. Ferguson, Alice Dawson].

1979-1985 Working papers on South American Indians, vol. I-V.

1984 (éd.), Marriage practices in Lowland South America, University of Illinois Press, Urbana/Chicago.

1991 The gift of birds. Featherwork of native South American peoples, Pennsylvania University Museum Publications, Philadelphia [ouvrage édité en collaboration avec Ruben E. Reina].

1993-1998 South American Indian studies, vol. I-VIII.

1994 The way real people ought to live: essays on the Peruvian Cashinahua, Waveland Press, Prospect Heights, Illinois.

\section{ARTICLES ET CHAPITRES D'OUVRageS}

1963 «The phonological hierarchy of Cashinahua (Pano)», in B. F. Olson (éd.), Studies in Peruvian Indian Languages I, SIL Publications in Linguistics and Related Fields, 9, University of Oklahoma, Norman, pp. 207-217.

1964 "General Summary of Research Findings », Información de Campo, 80 [includes: "Matrilocality and patrilineality in Cashinahua society », « Kinship and naming adjustments necessitated by incestuous unions among the Cashinahua »; spanish translation: Información de Campo, 385].

1965 «The Cashinahua of Southeastern Peru», Expedition, VII (4), pp. 4-9.

1967a «Change and the Cashinahua », Expedition, IX (2), pp. 4-8.

1967 « "Physical anthropology of the Cashinahua », Expedition, IX (2), pp. 9-15 [en collaboration avec Francis E. Johnston, Patricia S. Gindhart, Richard L. Jantz et Geoffrey F. Walker].

1969 «The population structure of the Peruvian Cashinahua: demographic, genetic, and cultural interrelationship ", Human Biology, XXXXI, pp. 29-41 [en collaboration avec Francis E. Johnston, Richard L. Jantz et Geoffrey F. Walker].

1973 «Banisteriopsis usage among the Peruvian Cashinahua », in Michael J. Harner (éd.), Hallucinogens and shamanism, Oxford University Press, New York, pp. 9-14 [in Kensinger, ibid., 1994, chap. 19]. 
1974 «Cashinahua medicine and medicine men », in Patricia Lyon (éd.), Native South Americans : ethnology of the least known continent, Little, Brown \& Co, Boston, pp. 282-288 [in Kensinger, ibid., 1994, chap. 18].

1975 «Studying the Cashinahua », in Jane Powell Dwyer (éd.), The Cashinahua of Eastern Peru, The Haffenreffer Museum of Anthropology, Brown University, Bristol, Rhode Island, pp. 9-85 [in Kensinger, ibid., 1994, chap. 1, 2 et 7].

1977 "Cashinahua notions of social time and social space », Acts of the 42nd International Congress of Americanists, II, pp. 223-244 [in Kensinger, ibid., 1994, chap. 10].

1980 The dialectics of person and self in Cashinahua society, presented at the American Anthropological Association symposium : "The body and the self : the concrete philosophy of person, self and society in Lowland South America », manuscript [in Kensinger, ibid., 1994, chap. 11].

1981 "Food taboos as markers of age categories in Cashinahua », in Kenneth M. Kensinger et Waud H. Kracke (éds), Food taboos in Lowland South America, Bennington College, Working Papers on South American Indians, Bennington, pp. 157-176 [in Kensinger, ibid., 1994, chap. 16].

1982 "Sex and food: reciprocity in Cashinahua society? », in Kenneth M. Kensinger (éd.), Sexual ideologies in lowland South America, Bennington College, Working papers on South American Indians, 5, Bennington, pp. 1-3.

1984 " An emic model of Cashinahua marriage », in Kenneth M. Kensinger (éd.), Marriage practices in Lowland South America, University of Illinois Press, Urbana/Chicago, pp. 221-251 [in Kensinger, ibid., 1994, chap. 9].

1985 "Cashinahua siblingship », in Judith Shapiro et Kenneth M. Kensinger (éds), The sibling relationship in Lowland South America, Bennington College, Working Papers on South American Indians, Bennington, pp. 20-24 [in Kensinger, ibid., 1994, chap. 8].

1986 «Panoan linguistic, folkloristic and ethnographic research : retrospect and prospect », in Harriet Klein et Louise Stark (éds), South American Indians languages: retrospect and prospect, Texas University Press, Austin, pp. 224-285 [preliminary version in América Indígena, XLIII (4), 1983].

1987 Bawdy rituals and Cashinahua stereotypes, presented at the American Anthropological Association symposium : " Gender rituals and the sexual self » [in Kensinger, ibid., 1994, chap. 4].

1988a «Invisible people: ostracism in Cashinahua society », in Richard R. Randolph, David M. Schneider et Mary M. Diaz (éds), Dialectics and gender, anthropological approaches. A festschrift for Robert and Yolanda Murphy, Westview Press, Boulder/Londres, pp. 170-179 [in Kensinger, ibid., 1994, chap. 15].

1988b Why bother: Cashinahua views of sexuality, presented at the American Anthropological Association symposium: "Anthropology Rediscovers Sex », manuscript [in Kensinger, ibid., 1994, chap. 6]. 
1989a «Hunting and male domination in Cashinahua society », in Susan Kent (éd.), Hunters as farmers. The implications of sedentism, Cambridge University Press, pp. 18-26 [in Kensinger, ibid., 1994, chap. 3].

1989b Hardfaced hussies and flacid fellows, presented at the American Anthropological Association symposium: "Shame on You! Shaming \& Social Control in Lowland South America », manuscript [in Kensinger, ibid., 1994, chap. 5].

1990a Living with spirit beings: tribal religion in Amazonia, manuscript [in Kensinger, ibid., 1994, chap. 17].

1990b "You killed my baby!": the dilemmas of medical intervention during fieldwork, presented at the American Anthropological Association symposium : "The Anthropologist as Healer », manuscript [in Kensinger, ibid., 1994, chap. 20].

1991a "Feathers make us beautiful: the meaning of Cashinahua feather headdresses ", in Ruben Reina et Kenneth M. Kensinger (éds), The gift of birds. Featherworking of Native South American Peoples, Pennsylvania University Museum Publications, Philadelphia, pp. 40-49 [in Kensinger, ibid., 1994, chap. 23].

1991b Panoan kinship terminology and social organization : dravidian or kariera, or something else?, presented at the 47th International Congress of Americanists symposium: "Classic Panoan topics in light of recent research », New Orleans, manuscript [in Kensinger, ibid., 1994, chap. 13].

1992a «The body knows, or, a body of knowledge », Expedition, XXXIII (3), pp. 37-45.

1992b Disposing of the dead, presented at the American Anthropological Association symposium : " Death, mourning and the afterlife in Lowland South America ", manuscript [also published in: The Latin American Anthropology Review, 1993, 5 (2), pp. 57-60 ; in Kensinger, ibid., 1994, chap. 21].

1993a «Leadership and factionalism in Cashinahua society », in Waud H. Kracke (éd.), Leadership in Lowland South America, Bennington College, South American Indian Studies, 1, Bennington, pp. 19-22 [originally written in 1974 ; in Kensinger, ibid., 1994, chap. 14].

1993b "When a turd floats by: Cashinahua metaphors of contact », in Terence Turner (éd.), Cosmology, values, and inter-ethnic contact in South America, Bennington College, South American Indian Studies, 2, Bennington, pp. 37-38 [in Kensinger, ibid., 1994, chap. 24].

1994 "The body knows, Cashinahua perspectives on knowledge », Acta Americana, II (2), pp. 7-14 [originally presented at the 48th International Congress of Americanists, Uppsala, Sweden, 1994 ; in Kensinger, ibid., 1994, chap. 22].

1997a «Cambio de perspectivas sobre las relaciones de género entre los Cashinahua desde 1955 a 1994 », in Michel Perrin et Marie Perruchon (éds), Complementariedad entre hombre y mujer, relaciones de género desde la perspectiva amerindia, Abya Yala, Quito, pp. 109-124.

1997b "An ethnographer's journey : belief, skepticism and a road to knowledge », Acta Americana, V (1), pp. 5-20. 
1998a «Changing perspectives on Cashinahua residential practices : 1955-1995 », in Debra Picchi (éd.), Unsettled communities: changing perspectives on South American Indigenous settlements, Bennington College, South American Indian Studies, 5, Bennington, pp. 29-32.

1998b "Los Cashinahua », in Federica Barclay et Fernando Santos Granero (éds), Guía etnográfica de la Alta Amazonía, vol. 3, Flacso/IFEA, Quito, pp. 1-124.

2002 "The dilemmas of co-paternity in Cashinahua society», in Stephen Beckermann et Paul Valentine (éds), Cultures of multiple fathers : the theory and practice of partible paternity in South America, University of Florida Press, Gainesville, pp. 14-26.

2003a «Being a real man : in memory of grompes », Tipití. Journal of the Society for the Anthropology of Lowland South America, I (1), pp. 99-112.

2003b «Satisfaction, ambivalence, nostalgia, and regrets : reflections on change in Cashinahua life, 1955-1997 », Acta Americana, XI (1), pp. 5-16.

\section{FILMOGRAPHIE}

Gray Gordon

2009 Understanding real people. Ken Kensinger's Cashinahua collection, Habitus Films, $54 \mathrm{mn}$ [film inédit, à partir d'entretiens avec Kensinger autour de la collection cashinahua qu'il avait donnée en 1966 au département d'anthropologie de Temple University].

Philippe ERIKSON

Université Paris Ouest Nanterre La Défense 